\title{
Crowd-anticrowd theory of the Minority Game
}

\author{
M. Hart, P. Jefferies and N.F. Johnson \\ Physics Department, Oxford University, Oxford, OX1 3PU, U.K. \\ P.M. Hui \\ Department of Physics, The Chinese University of Hong Kong, Shatin, \\ New Territories, Hong Kong
}

\begin{abstract}
The Minority Game is a simple yet highly non-trivial agent-based model for a complex adaptive system. Despite its importance, a quantitative explanation of the game's fluctuations which applies over the entire parameter range of interest has so far been lacking. We provide such a quantitative description based on the interplay between crowds of like-minded agents and their anti-correlated partners (anticrowds).
\end{abstract}

PACS: 01.75.+m, 02.50.Le, 05.40.+j, 87.23.Ge 
Agent-based models of complex adaptive systems are attracting significant interest across a variety of disciplines [1]. An important application currently receiving much attention within the physics community, is the study of fluctuations in financial markets [2]. Each agent knows the past ups and downs in the price index of a financial market and must decide how to trade based on this global information. These decisions then feed back to generate the fluctuations or 'volatility' of the price index itself. The resulting dynamical, many-body system is hence highly non-linear; indeed the combination of adaptability and frustration make such agent-based systems arguably richer and more challenging than those typically studied in condensed matter and statistical physics.

The Minority Game (MG) introduced by Challet and Zhang [3, [1], offers possibly the simplest paradigm for such a complex, adaptive system. The development of a complete, quantitative theory of the MG is of fundamental importance - the MG has therefore been the subject of intense research activity in the physics community [2 [10]. The most striking feature arising from numerical simulations [4] is the non-monotonic variation in the 'volatility' $\sigma$ (i.e. the standard deviation of the fluctuations) of the 'price' as the agents' memory-length $m$ is varied. Challet et al provided a sophisticated formal connection between the MG and spin glass systems [5] yielding many fascinating quantitative results for the MG's dynamics. However, no general theory has yet been provided which yields quantitative agreement with the numerical results for $\sigma$ [4] over the entire range of $m, s$ and $N$ values.

This paper shows that a theoretical model can be constructed in a surprisingly simple way by incorporating the 'crowd' effects (i.e. strong inter-agent correlations) which arise within the interacting, many-agent population. The results yield very good agreement with the numerical results for $\sigma$ over the entire range of $m, s$ and $N$. The non-monotonic behaviour of the volatility $\sigma$ [3, 1] results from a fascinating interplay between the actions of groups of like-minded agents ('crowds') and the actions of opposing groups ('anticrowds').

The MG [3] comprises an odd number of agents $N$ who choose repeatedly between option 0 (e.g. buy) and option 1 (e.g. sell). The winners are those in the minority group, e.g. sellers win if there is an excess of buyers. The outcome at each timestep represents the winning 
decision, 0 or 1 . A common bit-string of the $m$ most recent outcomes is made available to the agents at each timestep. The agents randomly pick $s$ strategies at the beginning of the game, with repetitions allowed. After each turn, the agent assigns one (virtual) point to each of his strategies which would have predicted the correct outcome. At each turn of the game, the agent uses the most successful strategy, i.e. the one with the most virtual points, among his $s$ strategies. The strategy-space $\mathcal{V}_{m}$ forms a $2^{m}$-dimensional hypercube with strategies at the $2^{2^{m}}$ vertices. Fortunately, the MG's standard deviation is essentially unchanged if a 'reduced' strategy space $\mathcal{U}_{m}$ is used instead of $\mathcal{V}_{m}$ [3]: the $\mathcal{U}_{m}$ only contains $2^{m+1}$ strategies or equivalently $2^{m}$ strategy pairs $\{\mathcal{G}\}$. The two strategies within a given pair $\mathcal{G}$ are anticorrelated, i.e. they differ by the maximum possible Hamming distance $d_{H}=2^{m}\left[3\right.$. Strategies between any two pairs $\mathcal{G}$ and $\mathcal{G}^{\prime}$ are uncorrelated, i.e. they differ by $d_{H}=2^{m-1}$. The results presented in this paper employ the reduced strategy space.

If $n_{R}$ agents use the same strategy $R$, then they will act as a 'crowd', i.e. they will make the same decision. If $n_{\bar{R}}$ agents simultaneously use the anticorrelated strategy $\bar{R}$, they will make the opposite decision and will hence act as an 'anticrowd' $(\mathcal{G} \equiv(R, \bar{R}))$. If $n_{R} \approx n_{\bar{R}}$ for all $\mathcal{G}$, then the actions of the crowds and anticrowds cancel and the standard deviation $\sigma$ of the number of agents $A(t)$ choosing a given option will be small. In contrast if $n_{R} \gg n_{\bar{R}}$ for all $\mathcal{G}$, then $\sigma$ will be large. Since there is no correlation between $\mathcal{G}$ and $\mathcal{G}^{\prime}$, each group $\mathcal{G}$ comprising a crowd-anticrowd pair $\left(n_{R}, n_{\bar{R}}\right)$ can be taken as contributing to $A(t)$ via a separate, essentially random walk in time of step-size $\left|n_{R}-n_{\bar{R}}\right|$. The variances of these walks can be summed to obtain the standard deviation of $A(t)$. Hence the MG standard deviation is given by

$$
\sigma=\left[\sum_{\mathcal{G}=1}^{2^{m}} \sigma_{\mathcal{G}}^{2}\right]^{\frac{1}{2}}=\left[\sum_{\mathcal{G} \equiv(R, \bar{R})} \frac{1}{4}\left|n_{R}-n_{\bar{R}}\right|^{2}\right]^{\frac{1}{2}}
$$

where both time-averaging, for a given configuration of initial strategies, and configurationaveraging have been carried out. We now demonstrate that this crowd-anticrowd cancellation underlies the numerical results for $\sigma$ vs. $m$ [1]. We run the numerical simulation of the MG and wait until transients in $A(t)$ have disappeared. At timestep $t_{0}$, we read out the 
number of players playing each strategy $R$, where $R=1,2, \ldots 2^{m+1}$. For each strategy pair $\mathcal{G}=(R, \bar{R})$, we obtain $n_{R}-n_{\bar{R}}$ at time $t_{0}$ and hence calculate $\sigma$. We then average this $\sigma$ over 1000 timesteps to simulate the time-averaging (our results are insensitive to the precise time-averaging procedure). Finally, we average over 16 runs to simulate the configurationaveraging. Figure 1 compares the resulting time and configuration-averaged $\sigma$ with that obtained from the numerical MG simulation. The agreement is very good for all $m, s$ and $N$ (not shown). Hence the crowd-anticrowd cancellation quantitatively explains the numerical results for $\sigma$ [4].

Since the labels $R$ are arbitrary in Eq. (1), the ordering of strategies $\left\{n_{R}\right\}$ has no particular significance. At any particular time $t_{0}$, these $2^{m+1}$ strategies can be ranked according to their virtual points by a sort-operation $\Theta$ acting on the list $\left\{n_{R}\right\}$. Hence $\left\{n_{R}\right\} \stackrel{\Theta}{\mapsto}$ $\left\{n_{\rho}\right\}$ where $\rho$ is the virtual-point rank label with $\rho=1$ being the highest scoring strategy and $\left\{n_{\rho}\right\} \equiv n_{\rho=1}, n_{\rho=2}, \ldots n_{\rho=2^{m+1}}$. Alternatively, strategies can be ranked according to their popularity $\left\{n_{r}\right\}$ where $\left\{n_{r}\right\} \equiv n_{r=1}, n_{r=2}, \ldots n_{r=2^{m+1}}$, i.e. strategy $r=1$ is that used by the largest number of agents, strategy $r=2$ is that used by the second largest number of agents, etc. Note that $\left\{n_{r}\right\}$ can be obtained from $\left\{n_{R}\right\}$ and hence $\left\{n_{\rho}\right\}$ by sort operations, i.e. $\left\{n_{R}\right\} \stackrel{\Psi}{\mapsto}\left\{n_{r}\right\}$ and hence $\left\{n_{\rho}\right\} \stackrel{\Gamma}{\mapsto}\left\{n_{r}\right\}$. Since each agent plays the available strategy having highest virtual points, it follows that

$$
n_{r}=N\left(\left[1-\frac{(r-1)}{2^{m+1}}\right]^{s}-\left[1-\frac{r}{2^{m+1}}\right]^{s}\right)
$$

where $\sum_{r=1}^{2^{m+1}} n_{r}=N$ as required. Agents are discrete objects; hence the simulation tends to produce discrete steps in the curves of $n_{r}$ as a function of $r$, particularly for large $m$ where the total number of strategies $2^{m+1}$ exceeds $N$. Hence we convert the theoretical $n_{r}$ values of Eq. (2) to an integer. For large $m$ such that $2^{m+1} \gg N$, the resulting theoretical values are typically $n_{r} \sim 1$ for small $r$ and $n_{r}=0$ for $r>N$. We have checked that the theoretical and numerical values for $n_{r}$ agree for general $m$.

For the virtual-point ordered list $\left\{n_{\rho}\right\}$, the strategy $\rho^{\prime}=2^{m+1}+1-\rho$ is always anticorrelated to the strategy $\rho$, i.e. $\rho^{\prime} \equiv \bar{\rho}$. Hence knowledge of the sort operation $\Gamma$ completely 
determines where each strategy's anticorrelated partner is located in the popularity-ordered list $\left\{n_{r}\right\}$. Since we are here only interested in time-averaged and run-averaged $\sigma$, we only need to consider the probability distribution of locations of $\bar{r}$ in the popularity-ordered list $\left\{n_{r}\right\}$. We therefore replace the sort operation $\Gamma$ by a probability function $P\left(r^{\prime}=\bar{r}\right)$ which gives the probability that any strategy $r^{\prime}$ is the anti-correlated partner of strategy $r$ in the list $\left\{n_{r}\right\}$. Hence Eq. (1) becomes

$$
\sigma=\left[\frac{1}{2} \sum_{r=1}^{2^{m+1}} \sum_{r^{\prime}=1}^{2^{m+1}} \frac{1}{4}\left|n_{r}-n_{r^{\prime}}\right|^{2} P\left(r^{\prime}=\bar{r}\right)\right]^{\frac{1}{2}} .
$$

When the virtual-point ordered list $\left\{n_{\rho}\right\}$ and the popularity-ordered list $\left\{n_{r}\right\}$ are identical, then $P\left(r^{\prime}=\bar{r}\right)$ will be a $\delta$-function at $r^{\prime}=2^{m+1}+1-r$ and hence Eq. (3) has the same form as Eq. (1). In the opposite case where the two ordered lists are uncorrelated, $P\left(r^{\prime}=\bar{r}\right)$ should be a flat distribution.

Figure 2 shows $P\left(r^{\prime}=\bar{r}\right)$ for $r=1$ as a function of $r^{\prime}$, taken from the numerical MG simulation at $m=2,5$ and 10 . For small $m(m=2)$ the anticorrelated strategy to the most popular strategy (i.e. $r=1$ ) is at $r^{\prime}=2^{m+1}$, i.e. it is the least popular strategy. Hence $P\left(r^{\prime}=\bar{r}\right)$ resembles the $\delta$-function limiting case mentioned above. Very few agents will therefore play this anticorrelated strategy. Hence the crowd-anticrowd cancellation will be small and $\sigma$ will be large, as can be seen in Fig. 1. As $m$ increases $(m=5)$ a remarkable effect occurs: the peak in $P\left(r^{\prime}=\bar{r}\right)$ moves up toward $r=1$. Hence both $r=1$ and its anticorrelated partner $\bar{r}$ are now very popular. For $m=2$ it seemed like there was an effective 'repulsion' between $r$ and $\bar{r}$; for $m=5$ this seems more like an attraction yielding a bound 'exciton-like' pair with the crowd (anticrowd) playing the role of the positive (negative) charge. For large $m(m=10)$, the ability of the anticrowd to 'screen' the crowd has decreased yielding a rather flat distribution. The strong crowd-anticrowd correlation which appears as $m$ increases means that the crowd and anticrowd become comparable in size. Hence $\sigma$ is small for $m \sim 5-6$, in agreement with Fig. 1. Note that the MG cannot fully 'optimize' itself by building equal-sized crowds and anticrowds because of the strategy-space frustration built in at the beginning of the game. In modified MG models such as Ref. 11 
which features evolving stochastic strategies, this frustration is able to relax - similar sized crowds and anti-crowds hence emerge yielding a smaller-than-random $\sigma$ for general $m$.

For small $m$, the two lists $\left\{n_{\rho}\right\}$ and $\left\{n_{r}\right\}$ will be very similar, hence $P\left(r^{\prime}=\bar{r}\right) \sim$ $\delta_{r^{\prime}, 2^{m+1}+1-r}$. The discreteness of the agents is now unimportant since $n_{r} \gg 1$, hence $n_{r}$ can be treated as continuous. Equations (2) and (3) yield

$$
\begin{aligned}
\sigma_{\text {low } m}= & \frac{N}{2}\left[\sum _ { r = 1 } ^ { 2 ^ { m } } \left[\left(1-\frac{r-1}{2^{m+1}}\right)^{s}-\left(1-\frac{r}{2^{m+1}}\right)^{s}\right.\right. \\
& \left.\left.-\left(\frac{r}{2^{m+1}}\right)^{s}+\left(\frac{r}{2^{m+1}}\right)^{s}\left(1-\frac{1}{r}\right)^{s}\right]^{2}\right]^{\frac{1}{2}} .
\end{aligned}
$$

For $s=2$ this becomes

$$
\sigma_{\text {low } m}=\frac{N}{\sqrt{3} 2^{\frac{m}{2}+1}}\left[1-2^{-2(m+1)}\right]^{\frac{1}{2}} .
$$

The inset in Fig. 3 shows these analytic curves for $s=2$ and $s=4$ (thick solid lines monotonically decreasing). As might be expected using the extreme $\delta$-function form for $P\left(r^{\prime}=\bar{r}\right)$, these curves produce upper estimates of $\sigma$ for small $m$. Now consider the opposite extreme of uncorrelated $r^{\prime}$ and $\bar{r}$, i.e. the flat distribution $P\left(r^{\prime}=\bar{r}\right) \sim 2^{-(m+1)}$. For $s=2$ this gives

$$
\sigma_{\text {low } m}=\frac{N}{\sqrt{3} 2^{\frac{(m+3)}{2}}}\left[1-2^{-2(m+1)}\right]^{\frac{1}{2}} .
$$

Equation (6), and its generalization for $s>2$, produce lower estimates (thin dashed lines in Fig. 3 inset). For a given $s$, the $\sigma$ values obtained from separate numerical runs tend to be scattered between the two corresponding limiting analytic curves. For larger $m(m>6)$ the discreteness of the agents implies that $n_{r} \sim 1$ for $r<N$ while $n_{r}=0$ for $r>N$. Using the integer form of Eq. (2) for high $m$, and the flat distribution for $P\left(r^{\prime}=\bar{r}\right)$, yields

$$
\sigma_{\text {high } m}=\frac{\sqrt{N}}{2}\left[1-\frac{N}{2^{m+1}}\right]^{\frac{1}{2}} .
$$

Hence $\sigma$ approaches the coin-toss limit from below as $m \rightarrow \infty$ (thick solid line monotonically increasing in Fig. 3 inset) as observed numerically. The analytic curves in Fig. 3 (inset) have further similarities with the numerical results of Fig. 1: (a) a minimum in $\sigma$ for 
$s=2$, (b) minimum moves to higher $m$ as $s$ increases, (c) minimum becomes shallower as $s$ increases, (d) $\sigma$ is not sensitive to $s$ for large $m$. Equation (7) also shows that $\sigma_{\text {high } m} \rightarrow 0$ at $N=2^{m+1}$, i.e. for $m \sim 5-6$. Using conditional probabilities to go one step beyond the approximation of a flat $P\left(r^{\prime}=\bar{r}\right)$ distribution, we find that $\sigma_{\text {high } m}$ for general $s$ is given by $\sqrt{N}\left[1-2^{-(m+1)}(N s-1)\right]^{\frac{1}{2}} / 2$. Interestingly, this form is consistent with the results of the spin-glass replica approach [12,13].

Figure 3 (main graph) shows the spread of numerical values for different runs compared to the full crowd-anticrowd theoretical calculation. The appropriate analytic expressions for the probability function $P\left(r^{\prime}=\bar{r}\right)$ in Eq. (3) involve multiple sums and are cumbersome: we therefore obtained the results for each $m$ in Fig. 3 by generating the corresponding $P\left(r^{\prime}=\bar{r}\right)$ similar to those in Fig. 2. The agreement is very good. The theoretical points tend to lie toward the high end of the numerical spread, for example at $m=2$; this can be attributed to the fact that our theory neglects accidental degeneracies in the virtual-point ordered list $\left\{n_{\rho}\right\}$. We have checked that including a stochastic (i.e. coin-toss) process to break such ties, reduces the theoretical $\sigma$ values down toward the mid-point of the numerical spread hence making the agreement even better [13].

In summary we have presented an analytic analysis of crowding effects in the MG which provides quantitative agreement with the main finding of Ref. [4] across the entire parameter range of interest.

We thank D. Challet for his comments. 


\section{REFERENCES}

[1] W.B. Arthur, Science 284, 107 (1999).

[2] See the Econophysics web-site http://www.unifr.ch/econophysics.

[3] D. Challet and Y.C. Zhang, Physica A 246, 407 (1997); ibid. 256, 514 (1998); ibid. 269, 30 (1999).

[4] R. Savit, R. Manuca and R. Riolo, Phys. Rev. Lett. 82, 2203 (1999).

[5] D. Challet and M. Marsili, Phys. Rev. E 60, R6271 (1999); D. Challet, M. Marsili, and R. Zecchina, Phys. Rev. Lett. 84, 1824 (2000); D. Challet and M Marsili, condmat/9908480.

[6] A. Cavagna, I. Giardina, J.P. Garrahan and D. Sherrington, Phys. Rev. Lett. 83, 4429 (1999).

[7] R. D'Hulst and G.J. Rodgers, Physica A 270, 514 (1999).

[8] A. Cavagna, Phys. Rev. E 59, R3783 (1999).

[9] N.F. Johnson, P.M. Hui, D. Zheng and M. Hart, J. Phys. A: Math. Gen. 32 L427 (1999).

[10] N.F. Johnson, M. Hart and P.M. Hui, Physica A 269, 1 (1999). This conference paper provides a preliminary report of the qualitative effects of crowding.

[11] N.F. Johnson, P.M. Hui, R. Jonson and T.S. Lo, Phys. Rev. Lett. 82, 3360 (1999).

[12] D. Challet, private communication.

[13] P. Jefferies, M. Hart, N.F. Johnson and P.M. Hui, forthcoming. 


\section{FIGURES}

FIG. 1. Standard deviation $\sigma$ for the Minority Game as a function of memory-size $m$ for $s=2,3,4$ strategies per agent and $N=101$ agents. Solid curve: numerical simulation. Dashed

curve: crowd-anticrowd theory using Eq. (1). Random (coin-toss) limit $\sigma=\sqrt{N} / 2=5.0$ is indicated.

FIG. 2. Probability function $P\left(r^{\prime}=\bar{r}\right)$ giving the probability that the strategy ranked $r^{\prime}$ on the popularity-ordered list, is anti-correlated with the strategy ranked $r$. Results are shown for $r=1$ (i.e. most popular strategy) as a function of $r^{\prime}$ for $m=2$ (dotted-dashed), $m=5$ (dotted) and $m=10$ (solid). $s=2$ and $N=101$. Note that $\sum_{r^{\prime}} P\left(r^{\prime}=\bar{r}\right)=1$.

FIG. 3. Theoretical crowd-anticrowd calculation (solid circles) vs. numerical simulations (open circles) for $s=2, N=101$. 16 numerical runs are shown for each $m$. Inset: Theoretical curves for $\sigma$ using limiting analytic forms in Eqs. (4)-(7). 


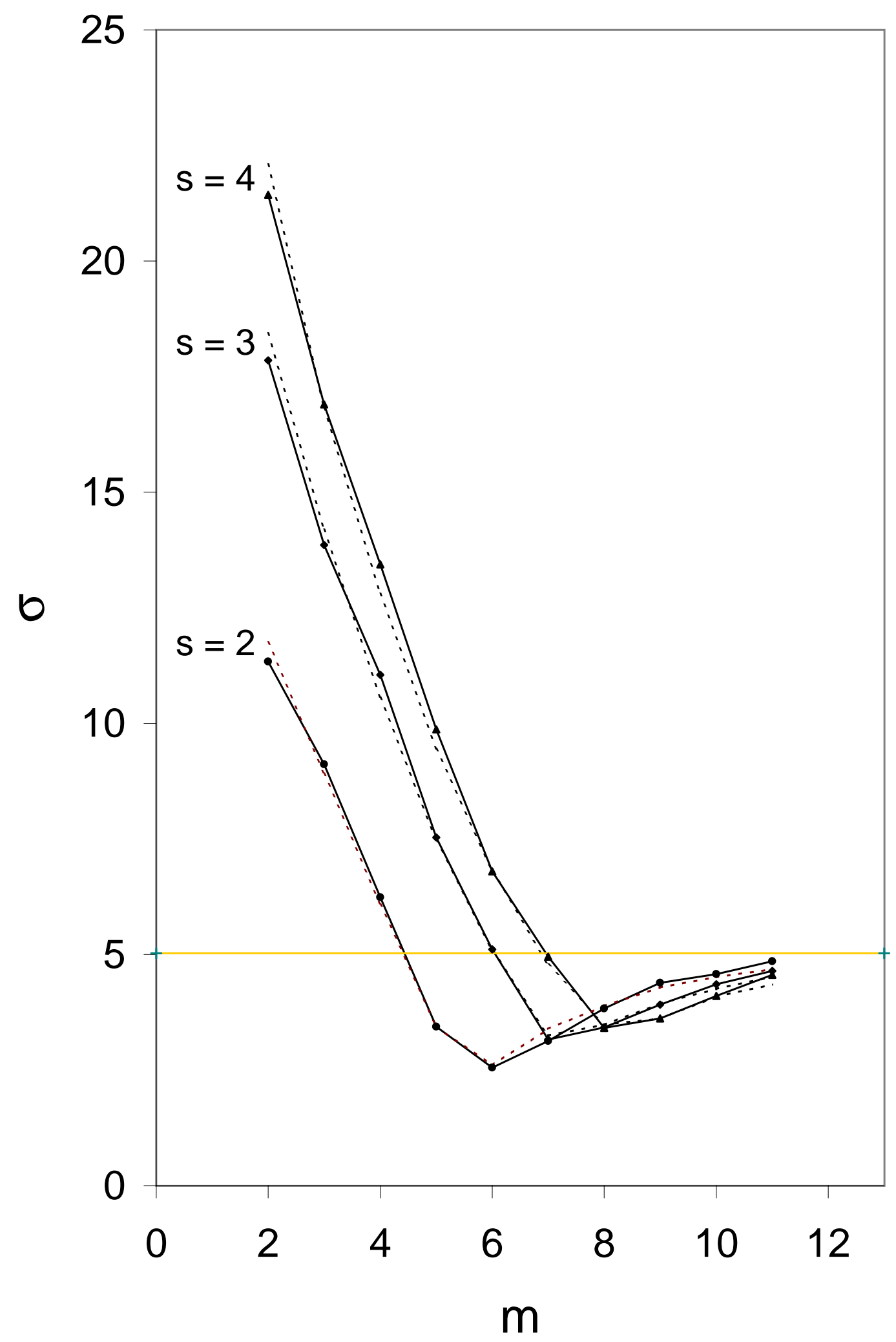




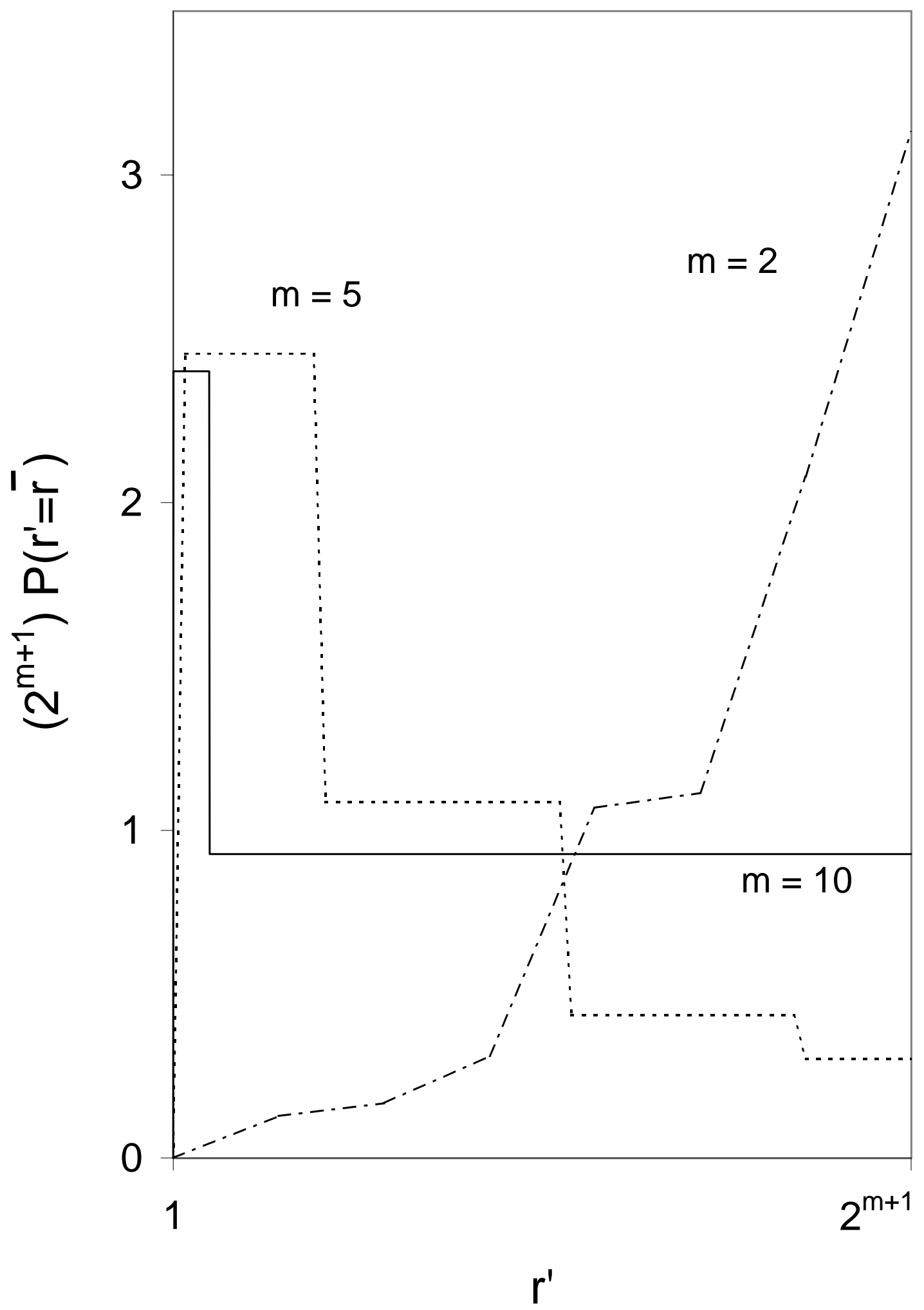




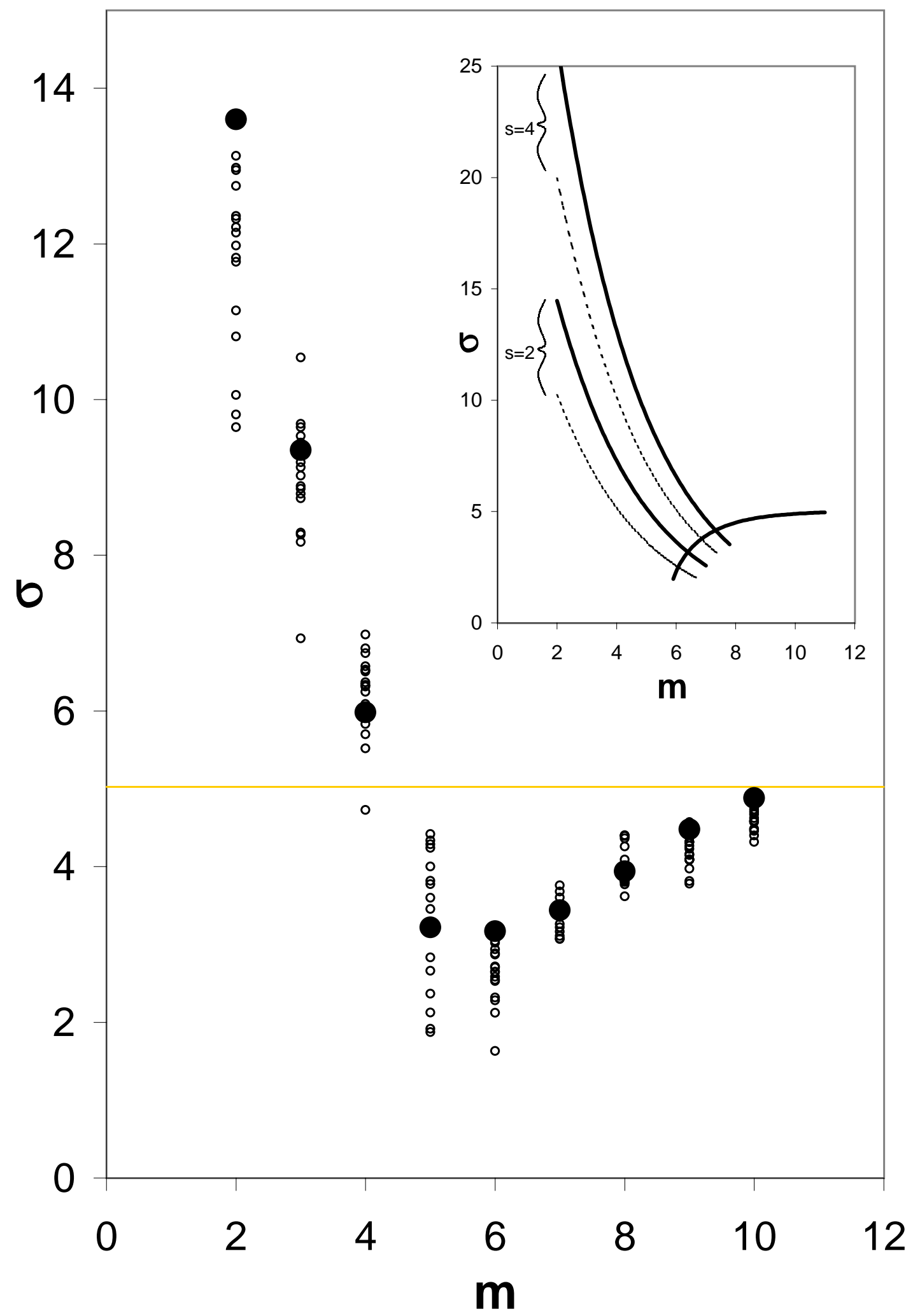

